\title{
Efeitos de curto prazo de um programa de imaginação sobre o medo de queda de indivíduos pós acidente vascular encefálico
}

\author{
Larissa Rebola Volpi da Silva ${ }^{1,2}$ \\ André Luiz Felix Rodacki ${ }^{1}$ \\ Vanessa Zadorosnei Rebutini ${ }^{1}$ \\ Suelen Meira Góes 1,2 \\ Ricardo Weigert Coelho ${ }^{2}$ \\ Joice Mara Facco Stefanello ${ }^{1,2}$ \\ ${ }^{1}$ Centro de Estudos em Comportamento Motor (CECOM), Programa de Pós Graduação em Educação Física, \\ Departamento de Educação Física, Setor de Ciências Biológicas, Universidade Federal do Paraná, Curitiba, \\ PR, Brasil \\ ${ }^{2}$ Laboratório de Psicofisiologia do Exercício e Esporte (LAPPES). Programa de Pós Graduação em \\ Educação Física, Departamento de Educação Física, Setor de Ciências Biológicas, Universidade Federal \\ do Paraná, Curitiba, PR, Brasil \\ Resumo: $O$ medo de cair é um dos principais problemas em indivíduos pós acidente vascular encefálico \\ (AVE). A imaginação pode ser uma experiência sucedida para diminuir este aspecto. O objetivo deste \\ estudo foi verificar o efeito de um programa de imaginação de curto prazo no medo de quedas em \\ indivíduos pós-AVE. Sete indivíduos acometidos por AVE participaram do estudo. A Escala Internacional de \\ Eficácia de Quedas (FES-I-Brasil) foi aplicada antes (PRÉ), depois (PÓS) e sete dias após a intervenção \\ (RET). Foram realizadas quatro sessões de familiarização e seis de prática da imaginação. Não houve \\ diferença significativa no escore total da FES-I-Brasil após intervenção $(p=0,46)$, exceto na tarefa \\ subir/descer escadas (PRÉ e PÓS, $p=0,04$; PRÉ e RET; $p=0,01$ ). Apesar da melhoria na tarefa subir/descer \\ escadas, alterações nos demais itens da escala não foram encontradas o que pode representar um efeito \\ relativamente reduzido do programa de imaginação de curto prazo.
}

Palavras-chave: Acidente vascular cerebral. Imaginação. Medo. Quedas.

\section{Short-term effects of imagery intervention on fear of falling in individuals post-stroke}

\begin{abstract}
The fear of falling is one of the main problems with subjects after stroke. Imagery could be one succeed experience to decrease this issue. The aim of this study was to verify the effect of a short-time imagery intervention in fear of falling in subjects after stroke. Seven individuals affected by stroke participated in the study. The Falls Efficacy Scale International (FES-I-Brazil) were used before (PRE), after (POST) and seven days after intervention (RET). Four session of familiarization and six of imagery were performed. There were no difference in FES-I-Brazil score after intervention $(p=0.46)$, except in up/down stairs task (PRE and POST, $p=0.04$; PRÉ and RET, $p=0.01$ ). Although, the improvement in up/down stairs task, no more changes were found in the scale items, which could represent a relatively reduced effect of short-time imagery intervention.
\end{abstract}

Keywords: Stroke. Imagery. Fear. Falls.

\section{Introdução}

O Acidente Vascular Encefálico (AVE) tem como consequências danos físicos, psico-afetivos e cognitivos relacionados com o local, extensão e natureza da lesão (BORGES, et al., 2007). Além dos déficits primários associados com o AVE, há um alto índice de complicações secundárias, nas quais incluem-se as quedas (BELGEN et al., 2006). Em pacientes que sofreram AVE, a incidência de queda pode chegar a $64,5 \%$ (BELGEN et al., 2006), o que constitui um índice mais elevado do que o observado nos idosos (45\%) entre 80 a 89 anos (CAMPBELL et al., 1981). Além de fraturas, lesões de tecidos moles, hospitalizações, imobilização, aumento da incapacidade, complicações médicas e risco de morte, o medo de cair é um dos principais problemas em indivíduos que sofreram AVE (BELGEN et al., 2006).

O medo de queda representa uma preocupação duradoura que se relaciona à perda de confiança no equilíbrio e na mobilidade, levando indivíduos acometidos pelo AVE a evitar atividades que poderiam ser capazes de realizar (TINETTI e WILLIAMS, 1998; CUMMING et al., 2000). Dessa forma, o medo de cair deixa de ser uma resposta protetora a uma ameaça real que impediria a execução de atividades com alto risco 
de quedas e de ferimentos em potencial, passando a restringir a realização de atividades que poderiam ser executadas com segurança. As consequências disso em relação à saúde social, mental e física das pessoas pode causar importante impacto (ZIJLSTRA et al., 2007).

O medo de cair não pode ser considerado apenas como uma consequência da queda em si, pois ele não é encontrado apenas entre aqueles que apresentam quedas recorrentes (HYNDMAN et al., 2002). As taxas de incidência do medo de queda em idosos variam de $29 \%$ a $92 \%$ para indivíduos com histórico recente e de $12 \%$ a $65 \%$ naqueles sem histórico. Nas pessoas que sofreram o AVE o medo de queda pode ser ainda maior, devido à diminuição do equilíbrio, força e mobilidade do indivíduo, que tem, como consequência, sua autoconfiança diminuída. Existem argumentos que os indivíduos que caem em decorrência dos sintomas iniciais do AVE podem estar mais propensos a temer novas quedas (

Além disso, o medo de cair também pode ocasionar quedas mesmo em pessoas idosas em geral que não tenham histórico de quedas. Indivíduos que limitam suas atividades por causa do medo de cair aumentam, particularmente, o risco de tornarem-se quedantes (FRIEDMAN et al., 2002). De fato, a diminuição da atividade física pode levar a um declínio funcional e aumentar o risco de quedas. Nesse sentido, o medo de cair pode estar associado à percepção da capacidade de realização de tarefas com sucesso. Assim, a autoeficácia e autoconfiança podem influenciar a capacidade de executar tarefas diárias com segurança, ou seja, com baixo risco de quedas. Pessoas com baixa autoeficácia e autoconfiança tendem a focar mais sobre suas limitações e deficiências (LOPES et al., 2009), o que pode alterar sua percepção do risco de quedas e aumentar sua incidência. Logo, a percepção do risco de quedas pode ser aumentada após AVE, onde um conjunto de limitações restringe a mobilidade e a capacidade de executar tarefas da vida diária.

Dessa forma, é importante promover uma melhor visão sobre o risco de queda, de maneira a proporcionar o desenvolvimento funcional dos indivíduos com segurança. A revisão sistemática realizada por Zijlstra et al. (2007) sobre as intervenções aplicadas para reduzir o medo de queda na população idosa, demonstrou que a experiência de realizar atividades com segurança pode levar a uma maior autoeficácia e a uma diminuição do medo de queda. Ziilstra et al. (2007) acreditam que a redução do medo de cair tem impacto positivo ao melhorar o bem-estar psicológico dos idosos. Além disso, observa-se um efeito benéfico para essses individuos que se reflete em melhorias no envolvimento e participação social e na manutenção ou aprendizagem de atividades da vida diária. Assim, a compreensão de estratégias que auxiliem na redução do medo de quedas em indivíduos acometidos pelo AVE, proporcionandoIhes maior autonomia e independência, assume grande relevância científica e social.

Nessa perspectiva, a prática da imaginação, além de ser utilizada como uma importante ferramenta psicológica para melhorar o desempenho motor funcional de indivíduos acometidos por AVE, tem sido bem sucedida na diminuição do risco de queda em outras populações. Hamel e Lajoie (2005) ${ }^{11}$ aplicaram a prática da imaginação em idosos durante uma tarefa dupla ("dual task"), que se relaciona com alto índice de quedas. Os resultados demonstraram que, após o treino usando a imaginação, os sujeitos automatizaram a manutenção da postura, o que permitiu direcionar a atenção para os fatores de risco do ambiente evitando assim as quedas. No contexto esportivo, a imaginação também tem contribuído para o controle do medo e para a melhora da autoconfiança em atletas (MAMASSIS e DOGANIS, 2004; $\underline{\text { COELHO et al., 2008; DUARTE }}$ et al., 2008).

Por ser uma experiência multisensorial, que ocorre na mente para recriar ou criar um evento sem a participação do ambiente (VEALEY, 1991), a imaginação pode ser uma experiência bem sucedida, pois contribui para a promoção dos níveis individuais de autoconfiança (STEFANELLO, 2007). Ver-se atuando bem em sua mente faz o indivíduo sentir que pode atuar sob circunstâncias adversas, especialmente naquelas acompanhadas do medo (WEINBERG e GOULD, 2001). Assim, a imaginação parece essencial para controlar as respostas físicas, mentais e comportamentais resultantes de situações causadoras de medo (DUARTE et al., 2008), as quais podem influenciar o medo de quedas. O presente estudo visou testar a hipótese de que um programa de intervenção de curto 
prazo baseado na imaginação pode diminuir o medo de queda em indivíduos pós-AVE.

\section{Métodos}

A presente investigação trata-se de um delineamento com testes pré e pós-tratamento de um grupo (THOMAS e NELSON, 2007), a fim de investigar o efeito de curto prazo de um programa de imaginação no medo de queda de indivíduos acometidos por AVE.
Um levantamento dos possíveis participantes do estudo em Hospitais, Clínicas, Associações e Centros de Reabilitação foi realizado. Nessa primeira busca, 148 voluntários aceitaram participar dos procedimentos do estudo, os quais foram aprovados pelo Comitê de Ética em Pesquisa da Secretaria da Saúde do Estado do Paraná (Parecer número 197/2010) atendendo as diretrizes propostas na Resolução 196/96 do Conselho Nacional de Saúde.

Quadro 1. Pontuação dos itens da National Institutes of Health Stroke Scale (NIHSS) determinante para exclusão dos participantes do estudo

\begin{tabular}{|c|c|c|}
\hline Itens & Domínios & Pontuação \\
\hline $1(\mathrm{a}, \mathrm{b}, \mathrm{c})$ & Nível de consciência & $\geq 1$ \\
\hline 3 & Visual & 3 \\
\hline 5 & Motricidade dos membros superiores & $\geq 3$ \\
\hline 6 & Motricidade dos membros inferiores & $\geq 3$ \\
\hline 7 & Ataxia & $\geq 1$ \\
\hline 8 & Sensibilidade & 2 \\
\hline 9 & Melhor linguagem & $\geq 1$ \\
\hline 10 & Disartria & $\geq 2$ \\
\hline 11 & Extinção ou inatenção & $\geq 1$ \\
\hline
\end{tabular}

Foram incluídos adultos de ambos os gêneros, com idade entre 26 e 65 anos, acometidos por AVE isquêmico ou hemorrágico confirmado por exame de imagem. Além disso, os voluntários deveriam ser capazes de adotar ortostatismo, permanecendo nesta postura com os pés paralelos e unidos, sem auxílio de terceiros e de realizar as atividades propostas de forma independente e segura. Foram excluídos indivíduos com distúrbios cognitivos que impedissem a compreensão/execução do programa de intervenção proposto (desorientação temporal/espacial, desatenção e alteração da memória operacional), verificados pelo Mini Exame do Estado Mental (FOLSTEIN et al., 1975), adotando-se os seguintes pontos de corte de acordo com os anos de escolaridade: analfabetos (20 pontos); de 1 a 4 anos (25 pontos); de 5 a 8 anos (27 pontos); de 9 a 11 anos (28 pontos) e indivíduos com escolaridade superior a 11 anos (29 pontos). Também foram excluídos os sujeitos com estado depressivo (acima de 20 pontos no Inventário de Depressão de Beck) (BECK et al., 1961). Para minimizar as diferenças em relação ao nível do comprometimento neurológico pós-AVE entre os participantes, foram padronizados os déficits motores e sensitivos e excluídos indivíduos inconscientes, desorientados, que não respondessem corretamente a comandos, cegos, atáxicos, afásicos, com disartria grave, e/ou que tivessem heminegligência, de acordo com a pontuação estipulada para os itens da National Institutes of Health Stroke Scale (NIHSS) (BROTT et al., 1989) (Quadro 1).

Sujeitos com comprometimento físico bilateral pós-AVE, episódios frequentes de tontura ou doenças vestibulares, ortopédicas e reumatológicas que pudessem comprometer a execução das atividades propostas também foram excluídos do estudo. Assim, após a aplicação dos critérios de inclusão e exclusão, 18 indivíduos foram selecionados, porém, dez alegaram dificuldade em comparecer à coleta de 
dados. Dessa forma, oito indivíduos iniciaram 0 tratamento experimental. Um sujeito não aderiu à pesquisa, devido à dificuldade em conseguir uma pessoa para acompanhá-lo até o local de realização do estudo e pela falta de conciliação com horários de outros tratamentos realizados. Assim, sete indivíduos participaram integralmente do presente estudo. A Figura 1 apresenta os procedimentos utilizados para a seleção dos participantes e o desenho experimental do estudo.

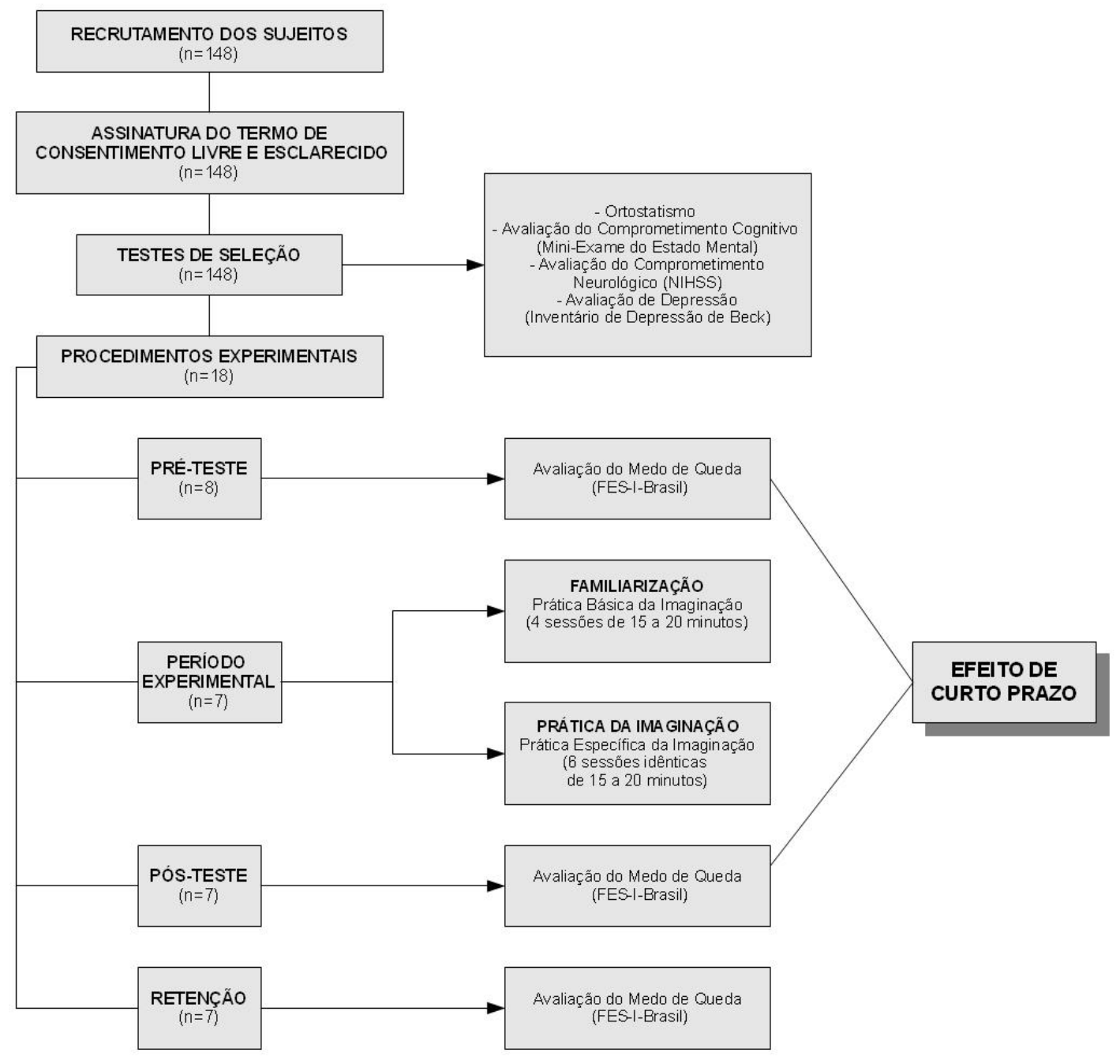

Figura 1. Desenho do estudo

Primeiramente, foram coletadas por meio de entrevista estruturada, as informações referentes aos dados pessoais, à topografia do comprometimento físico, ao tempo de lesão, à presença de patologias associadas, aos medicamentos e à incidência de quedas nos últimos seis meses. Posteriormente, os participantes foram avaliados sobre o medo de queda e submetidos a um programa de intervenção baseado na imaginação.

O medo de queda foi avaliado pela versão em português da Escala Internacional de Eficácia de Quedas (FES-I-Brasil), elaborada pelos membros do Prevention of Falls Network Europe (ProFaNE) (YARDLEY et al., 2005). A versão em português
(FES-I-Brasil) foi desenvolvida e validada por Camargos (2007) apresentando consistência interna $\alpha=0,93$, e confiabilidade de ICC $=0,84$ e 0,91 (intra e interexaminadores, respectivamente) (CAMARGOS, et al. 2010)

A FES-I-Brasil avalia o nível de preocupação do indivíduo em cair quando executa algumas atividades diárias, incluindo atividades externas e participação social. É um questionário de autorresposta composto de 16 itens. A resposta em cada item pode variar de 1 (sem preocupação) a 4 (muito preocupado) e o escore total de 16 a 64 pontos.

Para responder à FES-I- Brasil, os sujeitos foram orientados a marcar a alternativa que mais 
caracterizava o quão preocupados estavam com a possibilidade de uma queda durante a execução das atividades descritas na escala. Na hipótese da não realização de determinada atividade, os sujeitos foram orientados a responder a partir de sua percepção em realizá-la.

A avaliação do medo de queda dos indivíduos acometidos por AVE compreendeu três momentos: 24 a 48 horas antes do programa de intervenção (PRÉ), 24 a 48 horas após o término do programa de intervenção (PÓS) e sete dias após a conclusão da intervenção (RET). O PRÉ teve a finalidade de identificar a condição inicial dos participantes, enquanto o PÓS buscou identificar melhorias no medo de queda em função do programa de intervenção. O RET visou demonstrar se as melhorias obtidas durante a intervenção eram relativamente duradouras.

Antes de iniciar o programa específico de imaginação, foram realizadas quatro sessões que tiveram o objetivo de auxiliar os indivíduos no desenvolvimento de imagens vívidas (visuais e cinestésicas) e assim, fazerem uso da imaginação de forma mais eficaz - familiarização (WEINBERG e GOULD, 2001; STEFANELLO, 2007; STEFANELLO et al., 2010). Cada sessão teve duração de 15 a 20 minutos, com intervalo de um dia entre elas. Os exercícios imaginados consistiram em movimentos simples (um de membro superior, dois de membro inferior e um de tronco): bater o pé contra o chão, deslizar o pé no chão para frente e para trás, inclinar o tronco para os lados, levar uma das mãos até o ombro do lado oposto. Os praticantes permaneceram sentados confortavelmente em uma cadeira e receberam orientações sobre as atividades a serem realizadas, obedecendo à seguinte sequência para cada movimento: (a) observar a demonstração do movimento a ser imaginado; (b) executar fisicamente (uma única vez) o movimento demonstrado; (c) realizar um exercício de relaxamento orientado (de 2 a 3 minutos); (d) realizar a imaginação direcionada (primeiramente na perspectiva visual e depois na perspectiva cinestésica), com destaques para os pontos chaves da tarefa imaginada e (e) imaginar o movimento solicitado no mesmo ritmo da prática real.

Após o período de familiarização com a prática da imaginação, os participantes foram submetidos a um período de prática específica da imaginação, que foi composto por seis sessões idênticas de 15 a 20 min (três vezes semanais com intervalo de um dia entre cada sessão), obedecendo à mesma sequência de procedimentos empregados na familiarização. As tarefas incluíram atividades em ortostatismo relacionadas com a manutenção do equilíbrio, uma vez que o medo de queda relaciona-se à perda de confiança no equilíbrio (TINETTI e WILLIAMS, 1998; CUMMING et al., 2000). Como as situações que envolvem desafios do equilíbrio são, normalmente, as maiores causadoras do medo de queda e todas as atividades da vida diária avaliadas pelo questionário FES-I-BRASIL relacionam-se com 0 equilíbrio, as seguintes tarefas foram escolhidas para o período de treinamento: imaginar-se em pé, com os pés unidos e com os olhos abertos; imaginar-se em pé, com os pés unidos e com os olhos vendados; imaginar-se deslocando o centro de pressão até os limites de estabilidade, ou seja, inclinar o corpo, sem mudar a base de suporte, na direção anterior, posterior, para o lado direito e para o lado esquerdo; imaginar-se em pé sobre uma espuma. Os participantes foram orientados a imaginar as tarefas como se estivem visualizando a si mesmo (perspectiva visual) e como se estivessem sentindo os movimentos realmente (perspectiva cinestésica) de forma tranquila e com sucesso, sem temor. Todas as sessões (familiarização e da prática específica da imaginação) foram conduzidas de maneira individualizada.

Em função do número reduzido de participantes, análises não paramétricas foram adotadas. Assim, a estatística descritiva refere-se à mediana \pm desvio padrão. Para determinar as alterações com relação ao medo de queda nos indivíduos pós-AVE, após as seis sessões de prática da imaginação e na retenção, foi utilizado o teste Friedman ANOVA. Após a análise de variância de Friedman foi utilizado como "post hoc" o teste sugerido por Conover (1999). Todos os dados foram analisados no pacote Statistica (Statsoft, versão 7) e o nível de significância foi de $p<0.05$.

\section{Resultados}

As características físicas e as principais variáveis empregadas para descrever a amostra encontram-se na Tabela 1. A maior parte do grupo era composta por homens $(57,1 \%)$ e a maioria dos participantes havia sido acometida por Acidente Vascular Encefálico (AVE) do tipo 
isquêmico $(71,4 \%)$. Com relação à topografia do comprometimento físico pós-AVE, $57,1 \%$ dos participantes apresentavam hemiparesia esquerda e $42,9 \%$ hemiparesia direita. Apesar de $28,6 \%$ dos participantes não apresentar outras patologias associadas, 42,9\% apresentava hipertensão arterial sistêmica (HAS) e 28,6\% HAS e diabetes diagnosticadas. Constatou-se que dois dos sete participantes $(28,6 \%)$ haviam caído ao menos uma vez nos últimos seis meses.

Tabela 1. Características gerais da amostra

\begin{tabular}{lc}
\hline & Mediana \pm Desvio Padrão \\
\hline Idade (anos) & $55 \pm 14,38$ \\
N $^{\circ}$ AVE (unidade) & $1 \pm 0,53$ \\
Tempo de lesão (meses) & $13 \pm 27,97$ \\
NIHSS (score) & $3 \pm 1,15$ \\
MEM (score) & $29 \pm 2,06$ \\
BECK (score) & $12 \pm 5,29$ \\
\hline
\end{tabular}

$n^{\circ}$ AVE: número de Acidente Vascular Encefálico; NIHSS: Escala de Acidente Vascular Encefálico dos Institutos Nacionais de Saúde; MEM: Mini Exame do Estado Mental; BECK: Inventário de Depressão de Beck

Apesar de não terem sido encontradas diferenças significativas no escore total da Escala Internacional de Eficácia de Quedas (FES-I-Brasil) após o programa de intervenção $(p=0,46)$, foram observadas diferenças na tarefa de subir ou descer escadas $(p=0,04)$, quando os itens da escala foram considerados isoladamente, comparando PRÉ, PÓS e RET. O "post hoc" apontou diferenças para este item entre o PRÉ e o PÓS $(p=0,04)$ e entre PRÉ e RET $(p=0,01)$, mas que não se repetiram entre PÓS e RET $(p=0,43)$. No pré-teste, todos os indivíduos apresentavam preocupação com relação à queda (pontuação total acima de 16 pontos), destacando-se que o item subir ou descer escadas (item 7) ficou entre os itens com pontuação mais elevada no PRÉ: caminhar sobre superfície irregular $(4 \pm 0,79)$; andar em lugares cheios de gente $(4 \pm 0,95)$; subir ou descer uma ladeira $(4 \pm 1,41)$; pegar algo acima da cabeça ou do chão $(3 \pm 1,11)$; subir e descer escadas $(2 \pm 0,79)$ e andar sobre superfície escorregadia $(2 \pm 0,95)$.

\section{Discussão}

A preocupação em cair identificada no préteste, em todos os indivíduos que participaram do estudo (pontuação acima de 16 pontos), pode ser entendida pelo impacto causado pelo AVE. A diminuição do equilíbrio, da força, da mobilidade e da autoconfiança tem sido reportada em sujeitos com AVE (SCHMID e RITTMAN, 2007), confirmando que o medo de cair pode estar presente mesmo naqueles indivíduos que não têm histórico de quedas (HYNDMAN et al., 2002), visto que apenas dois participantes relataram ter caído nos últimos 6 meses.

Os itens da escala que apresentaram as maiores pontuações (caminhar sobre superfície irregular, andar em lugares cheios de gente, subir ou descer uma ladeira, pegar algo acima da cabeça ou do chão, subir ou descer escadas e andar sobre superfície escorregadia), indicando preocupação com as quedas durante a realização das atividades avaliadas, corroboram o estudo realizado por Lopes et al. (2009) com idosos utilizando a FES-I-Brasil. As pontuações mais altas foram encontradas em situações desafiadoras para o controle do equilíbrio e estão presentes em ambientes extra-domiciliares. Sendo assim, uma alta pontuação nestes itens pode indicar uma restrição de participação dos indivíduos em atividades sociais ou externas. Logo, parece que o risco de quedas futuras é maior quando os idosos com medo de cair limitam suas atividades (MARTIN et al., 2005).

O medo de queda elevado durante a subida e descida de escadas pode ser explicado pela complexidade da tarefa, a qual se constitui numa atividade difícil e ameaçadora e que tem sido associada à elevada incidência de queda (VERGHESE et al., 2008) e consequente mortalidade (TOSCANO e WINDAU, 1993). Dentre as mortes ocasionadas por quedas, a grande maioria $(\sim 85 \%)$ foi registrada durante a transposição de pisos em diferentes níveis, como rampas, degraus e escadas. Dessa forma, a transposição de escadas pode ser vista como um desafio para pessoas que sofreram AVE em 
função de suas limitações físicas e das diferenças de requisição entre a locomoção em nível plano e a escalada dos degraus (HERMAN et al., 2009). Subir e descer escadas apresenta maior variação nas curvas de Força de Reação do Solo (FRS), o que indica variabilidade na execução do movimento (RIENER et al., 2002; STACOFF et al., 2005). Deve-se, também, considerar as diferenças existentes com relação às características de contração (excêntrica/concêntrica) presentes nas ações de plano inclinado, que resultam em maior flexão das articulações de membro inferior na subida e maior grau de frenagem na descida em relação ao plano. Isso ocasiona um aumento do nível de exigência na execução da tarefa e consequente aumento do medo, em virtude da baixa autoeficácia para execução na subida ou do aumento da instabilidade na descida (RIENER et al., 2002). Além disso, as características físicas do executante também podem ser determinantes sobre o medo de queda, ou seja, quanto maior o comprometimento motor do indivíduo em função do AVE, menores serão suas capacidades funcionais o que resultará em menor autoeficácia e maior medo de quedas nessa situação (REEVES et al., 2009).

Há indícios que populações com limitações físicas similares às dos pacientes com AVE (idosos, por exemplo), passam a utilizar o corrimão ao subir e descer degraus (VERGHESE et al., 2008), principalmente devido à falta de confiança e baixa autoeficácia na realização dessas atividades. O medo e a baixa autoeficácia em subir e descer escadas tem sido, frequentemente, relacionados à falta de confiança sobre as capacidades motoras e ao próprio sucesso da realização da tarefa (VERGHESE et al., 2008). De fato, subir e descer escadas está entre as cinco atividades de vida diária que os idosos consideram mais difíceis de executar, especialmente devido às alterações físicas que ocorrem em consequência do envelhecimento (WILLIAMSON e FRIED, 1996; STARTZELL et al., 2000), acentuando o medo de queda nessa população (FREITAS e SCHEICHER, 2008; LOPES et al., 2009).

Existem argumentos de que o medo de queda tem relação com representações mentais equivocadas da execução do movimento, desencadeando o medo das consequências de execuções não eficientes de determinadas tarefas (VERGHESE et al., 2008). Dessa forma, os indivíduos devem possuir habilidades e capacidades para responder às demandas da tarefa e confiança de que podem solucioná-la. Logo, a melhora encontrada no presente estudo com relação ao medo de quedas referente à ação de subir ou descer escadas após o programa de intervenção baseado na imaginação pode ser atribuído à possível melhoria na autoconfiança dos participantes do estudo e, consequentemente, na percepção de eficácia.

A melhoria encontrada em um dos itens da FES-I Brasil em função do programa de imaginação pode estar relacionada com a experiência de visualizar-se realizando determinada tarefa com sucesso. Nessas condições é possível construir referências que possibilitam a posterior execução correta do movimento e reduzem o medo (WEINBERG e GOULD, 2001). O treinamento baseado na imaginação tem o propósito de fazer com que os indivíduos executem mentalmente uma tarefa, mesmo que algumas dificuldades não permitam sua materialização. Tal estratégia é utilizada em atletas com o intuito de prepará-los física, coordenativa e emocionalmente para algumas tarefas, possibilitando encoraja-los para execuções de movimentos considerados complexos e/ou perigosos como saltos mortais na ginástica, golpes específicos de luta, ações em modalidades de alto risco (GOUVEIA, 2001). Semelhante aos resultados encontrados com os atletas, houve relação entre o treinamento mental baseado na imaginação e a execução de tarefas com ações que desencadeavam medo, ou seja, movimentos que antes eram executados com restrições, tornaram-se classificáveis dentro de uma categoria de maior conforto após a intervenção da imaginação.

É importante ressaltar que o presente estudo mostrou algumas limitações, incluindo a dificuldade de padronização do comprometimento físico, cognitivo e psicológico pós-AVE (critérios de inclusão e de exclusão adotados), bem como a dificuldade de adesão dos participantes ao programa proposto, que refletiram no número reduzido de sujeitos. O número reduzido de sujeitos inviabilizou contemplar grupo controle e aleatorização dos participantes. Apesar da melhoria específica na tarefa de subir e descer escadas, não foram encontradas alterações nos demais itens da escala o que pode representar um efeito relativamente reduzido do programa de imaginação sobre outros componentes das 
atividades da vida diária analisadas no presente estudo. Assim, sugere-se que um maior tempo de intervenção, associado aos exercícios para a recuperação das capacidades físicas, possa trazer ainda mais benefícios para esta população com relação à diminuição do medo de queda. Propõe-se também que próximos estudos, contemplem maior número de sujeitos e incluam medidas de avaliação de equilíbrio e outros testes para verificar a funcionalidade e qualidade de vida dos indivíduos.

\section{Referências}

BECK, A. T. et al. An inventory for measuring depression. Archives of general psychiatry, United States, v. 4, n. 6, p. 561-571, 1961.

BELGEN, B. et al. The association of balance capacity and falls self-efficacy with history of falling in community-dwelling people with chronic stroke. Archives of Physical Medicine and Rehabilitation, Chicago, v. 87, n. 4, p. 554-561, 2006.

BORGES, D. et al. Fisioterapia - aspectos clínicos e práticos da reabilitação. São Paulo: 2007.

BROTT, T. et al. Measurements of acute cerebral infarction: A clinical examination scale. Stroke, New York, v. 20, n. 7, p. 864-870, 1989.

CAMARGOS, F. F. O. Adaptação transcultural e avaliação das propriedades psicométricas da falls efficacy scale-international: um instrumento para avaliar medo de cair em idosos. 2007. dissertação (Mestrado). Escola de Educação Física, Fisioterapia e Terapia Ocupacional - Programa de Pós Graduação em Ciências da Reabilitação, Universidade Federal de Minas Gerais, Belo Horizonte.

CAMARGOS, F. F. O. et al. Cross-cultural adaptation and evaluation of the psychometric properties of the Falls Efficacy Scale -

International Among Elderly Brazilians (FES-IBRAZIL). Adaptação transcultural e avaliação das propriedades psicométricas da Falls Efficacy Scale - International em idosos brasileiros (FES-I-BRASIL), Revista Brasileira de Fisioterapia, São Carlos, v. 14, n. 3, p. 237-243, 2010.

CAMPBELL, A. J. et al. Falls in old age: A study of frequency and related clinical factors. Age and Ageing, London, v. 10, n. 4, p. 264-270, 1981. I

COELHO, R. W. et al. O efeito da imaginação no desempenho e na precisão do saque no tênis de campo. Revista Brasileira de Cineantropometria e Desempenho Humano, Florianópolis, v. 10, n. 2, p. 176-183 2008.
CONOVER, W. J. Practical nonparametric statistics. 3‥ Ed. John Wiley \& Sons, United States, 1999.

CUMMING, R. G. et al. Prospective study of the impact of fear of falling on activities of daily living, SF-36 scores, and nursing home admission. Journals of Gerontology - Series A Biological Sciences and Medical Sciences, Washington, v. 55, n. 5, p. M299-M305, 2000.

DUARTE, L. H. et al. O medo na ginástica artística: treinamento e competição. Revista Mackenzie de Educação Física e Esporte, São Paulo, v. 7, n. 2, p. 51-67, 2008.

FOLSTEIN, M. F.; FOLSTEIN, S. E.; MCHUGH, P. R. 'Mini mental state'. A practical method for grading the cognitive state of patients for the clinician. Journal of Psychiatric Research, Oxford, v. 12, n. 3, p. 189-198, 1975.

FREITAS, M. A. V.; SCHEICHER, M. E. Preocupação de idosos em relação a quedas. Revista Brasileira de Geriatria e Gerontologia, Rio de Janeiro, v. 11, n. 1, p. 57-64, 2008.

FRIEDMAN, S. M. et al. Falls and fear of falling: Which comes first? A longitudinal prediction model suggests strategies for primary and secondary prevention. Journal of the American Geriatrics Society, New York, v. 50, n. 8, p. 1329-1335, 2002.

GOUVEIA, M. J. Tendências da investigação na psicologia do desporto, exercício e actividade física. Análise Psicológica, Lisboa, v. 1, n. XIX, p. 5-14, 2001.

HAMEL, M. F.; LAJOIE, Y. Mental imagery. Effects on static balance and attentional demands of the elderly. Aging - Clinical and Experimental Research, Milano, v. 17, n. 3, p. 223-228, 2005.

HERMAN, T. et al. The Dynamic Gait Index in healthy older adults: The role of stair climbing, fear of falling and gender. Gait and Posture, Oxford, v. 29, n. 2, p. 237-241, 2009 ..

HYNDMAN, D.; ASHBURN, A.; STACK, E. Fall events among people with stroke living in the community: Circumstances of falls and characteristics of fallers. Archives of Physical Medicine and Rehabilitation, Chigaco, v. 83, n. 2, p. 165-170, 2002.

LOPES, K. et al. Prevalência do medo de cair em uma população de idosos da comunidade e sua correlação com mobilidade, equilíbrio dinâmico, risco e histórico de quedas. Revista Brasileira de Fisioterapia, São Carlos, v. 13, n. 3 , p. 223-229, 2009..

MAMASSIS, G.; DOGANIS, G. The effects of a mental training program on juniors pre-competitive anxiety, self-confidence, and tennis performance. 
Journal of Applied Sport Psychology, Chapel Hill, v. 16, n. 2, p. 118-137, 2004.

MARTIN, F. C. et al. Fear of falling limiting activity in young-old women is associated with reduced functional mobility rather than psychological factors. Age and Ageing, London, v. 34, n. 3, p. 281-287, 2005.

REEVES, N. D. et al. Older adults employ alternative strategies to operate within their maximum capabilities when ascending stairs. Journal of Electromyography and Kinesiology, New York, v. 19, n. 2, p. e57-e68, 2009.

RIENER, R.; RABUFFETTI, M.; FRIGO, C. Stair ascent and descent at different inclinations. Gait and Posture, Oxford, v. 15, n. 1, p. 32-44, 2002.

SCHMID, A. A.; RITTMAN, M. Fear of falling: An emerging issue after stroke. Topics in Stroke Rehabilitation, Frederick, v. 14, n. 5, p. 46-55, 2007.

STACOFF, A. et al. Ground reaction forces on stairs: Effects of stair inclination and age. Gait and Posture, Oxford, v. 21, n. 1, p. 24-38, 2005.

STARTZELL, J. K. et al. Stair negotiation in older people: A review. Journal of the American

Geriatrics Society, New York, v. 48, n. 5, p. 567580, 2000.

STEFANELLO, J. M. F. Treinamento de competências psicológicas: em busca da excelência esportiva. São Paulo: Manole, 2007.

STEFANELLO, J. M. F.; MARQUES, C. P.; RODACKI, A. L. F. Assessment of motor imagery ability and training. Avaliação da capacidade e treinabilidade da imaginação motora. Revista Brasileira de Cineantropometria e Desempenho Humano, Florianópolis, v. 12, n. 6, p. 395-400, 2010.

THOMAS, J. R.; NELSON, J. K. Métodos de pesquisa em atividade física. $5^{\circ}$ Ed. Porto Alegre: Artmed, 2007.

TINETTI, M. E.; WILLIAMS, C. S. The effect of falls and fall injuries on functioning in communitydwelling older persons. Journals of Gerontology - Series A Biological Sciences and Medical Sciences, Washington, v. 53, n. 2, p. M112M119, 1998.

TOSCANO, G.; WINDAU, J. Fatal Work Injuries: Results from the 1992 National Census. Monthly Labor Review, Columbia, v. 116, n.1, p. 3-8, 1993. Disponível em: $<$ http://stats.bls.gov/OPUB/MLR/1993/10/art3full.p df >. Acesso em: 15/07/2011

VEALEY, R. S. Entrenamiento en imaginación para el perfeccionamiento de la ejecución. In: WILLIAMS, J. M. (Ed.). Psicología aplicada al deporte. Madrid: Bibilioteca Nueva, 1991. p.305338.

VERGHESE, J. et al. Self-Reported Difficulty in Climbing Up or Down Stairs in Nondisabled Elderly. Archives of Physical Medicine and Rehabilitation, Chicago, v. 89, n. 1, p. 100-104, 2008..

WEINBERG, R. S.; GOULD, D. Fundamentos da psicologia do esporte e do exercício. $2^{2}$. Ed. Artmed, Porto Alegre, 2001.

WILLIAMSON, J. D.; FRIED, L. P. Characterization of older adults who attribute functional decrements to 'old age'. Journal of the American Geriatrics Society, New York, v. 44, n. 12, p. 1429-1434, 1996.

YARDLEY, L. et al. Development and initial validation of the Falls Efficacy Scale-International (FES-I). Age and Ageing, London, v. 34, n. 6, p. 614-619, 2005.

ZIJLSTRA, G. A. R. et al. Interventions to reduce fear of falling in community-living older people: $A$ systematic review. Journal of the American Geriatrics Society, New York, v. 55, n. 4, p. 603615, 2007.

\section{Endereço:}

Larissa Rebola Volpi da Silva

Departamento de Educação Física da

Universidade Federal do Paraná

R. Coração de Maria, 92, BR116, Km 95 Jd.

Botânico

Curitiba PR Brasil

80215-370

Telefone: (41) 3360-4326

Fax: (41) 3360-4336

e-mail: larissa_ela@hotmail.com

Recebido em: 30 de setembro de 2011.

Aceito em:12 de dezembro de 2012.

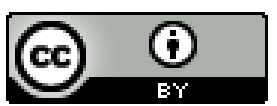

Motriz. Revista de Educação Física. UNESP, Rio Claro, SP, Brasil - elSSN: 1980-6574 - está licenciada sob Creative Commons - Atribuição 3.0 